\title{
Modelagem matemática das curvas de secagem e coeficiente de difusão de grãos de feijão-caupi (Vigna unguiculata (L.) Walp.) ${ }^{1}$
}

\author{
Mathematical modeling of the drying curves and diffusion coefficient of cowpea \\ grains (Vigna unguiculata (L.) Walp.)
}

\author{
Simone Janaína da Silva Morais ${ }^{2 *}$, Ivano Alessandro Devilla ${ }^{3}$, Daiane Assis Ferreira ${ }^{4}$ e Itamar Rosa Teixeira ${ }^{3}$
}

\begin{abstract}
RESUMO - Neste trabalho objetivou-se estudar a cinética de secagem do feijão-caupi (Vigna unguiculata (L.) Walp.), ajustar diferentes modelos matemáticos aos valores experimentais em função do teor de água e, determinar o coeficiente de difusão e a energia de ativação dos grãos durante a secagem. Os grãos de feijão-caupi foram submetidos à secagem sob as temperaturas de $25 ; 35 ; 45$ e $55 \pm 1{ }^{\circ} \mathrm{C}$ em secador experimental até o teor de água de aproximadamente $13 \%$ b.s. Realizou-se uma análise de regressão não linear para o ajuste de 12 modelos matemáticos aos dados experimentais de secagem. O coeficiente de difusão efetivo foi obtido por meio do ajuste do modelo matemático da difusão líquida. A variação do coeficiente de difusão de acordo com a temperatura de secagem foi analisada pelo modelo de Arrhenius, o qual permitiu a determinação da energia de ativação. O modelo de Henderson e Pabis Modificada foi o que melhor representou o processo de secagem do feijão-caupi. O coeficiente de difusão apresentou valores entre $8,84 \times 10^{-8} \mathrm{e}$ $20,17 \times 10^{-8} \mathrm{~m}^{2} \mathrm{~s}^{-1}$. A relação do coeficiente de difusão com a temperatura de secagem pode ser descrita pela equação de Arrhenius, apresentando uma energia de ativação de $27,16 \mathrm{~kJ} \mathrm{~mol}^{-1}$.
\end{abstract}

Palavras-chave: Cinética de secagem. Grãos-secagem. Grãos-armazenamento.

\begin{abstract}
This work aimed to study the drying kinetics of the cowpea (Vigna unguiculata (L.) Walp.), adjust different mathematical models to the experimental values as a function of water content, and determine the diffusion coefficient and the activation energy of the grains during drying. The cowpea grains were dried at temperatures of $25 ; 35 ; 45$ and $55 \pm 1{ }^{\circ} \mathrm{C}$ in an experimental dryer to a water content of approximately $13 \% \mathrm{db}$. A nonlinear regression analysis was carried out to adjust 12 mathematical models to the experimental drying-data. The effective diffusion coefficient was obtained by adjusting the mathematical model of liquid diffusion. The variance of the diffusion coefficient according to the drying temperature was analysed by the Arrhenius equation, which allowed determination of the activation energy. The Henderson and Modified Pabis model best represented the drying process of the cowpea. The diffusion coefficient presented values of between $8.84 \times 10^{-8}$ and $20.17 \times 10^{-8} \mathrm{~m}^{2} \mathrm{~s}^{-1}$. The ratio of the diffusion coefficient to the drying temperature can be described by the Arrhenius equation, having an activation energy of $27.16 \mathrm{~kJ} \mathrm{~mol}^{-1}$.
\end{abstract}

Key words: Drying kinetics. Grain-drying. Grain-storage.

\footnotetext{
*Autor para correspondência

${ }^{1}$ Recebido para publicação em 26/09/2011; aprovado em 30/12/2012

Parte da Dissertação de Mestrado do primeiro autor do Programa de Pós-Graduação em Engenharia Agrícola/UEG

${ }^{2}$ Departamento de Agronômia, Centro Universitário de Anápolis-Unievangélica, Anápolis-GO, Brasil, sijanaina@yahoo.com.br

${ }^{3}$ Departamento de Engenharia Agrícola, Unidade de Ciências Exatas e Tecnológicas, Universidade Estadual de Goiás/UEG, devilla@ueg.br, itamar.texeira@ueg.br

${ }^{4}$ Departamento de Engenharia Civil, Pontifícia Universidade Católica de Goiás/PUC Goiás, daianeagricola@gmail.com
} 


\section{INTRODUÇÃO}

O feijão-caupi (Vigna unguiculata (L.) Walp.), representa atualmente $20 \%$ da produção de feijão no Brasil (BORÉM; CARNEIRO, 2006). Pelo seu valor nutritivo, é cultivado principalmente para a produção de grãos, secos ou verdes, visando o consumo humano in natura, na forma de conserva ou desidratado. A conservação pela secagem baseia-se no fato de que tanto os microrganismos como as enzimas e todo o mecanismo metabólico necessitam de água para suas atividades. Com a redução da quantidade de água disponível até níveis seguros para armazenagem, serão reduzidos a atividade de água, a velocidade das reações químicas no produto e o desenvolvimento de micro-organismos (DOYMAZ, 2007).

Os modelos de cálculo da cinética de secagem são aplicados de modo diferente, dependendo do período considerado. Na literatura vários modelos foram propostos para analisar a secagem de produtos higroscópicos (MARTINAZZO et al., 2007).

$\mathrm{O}$ estudo e a modelagem matemática da cinética de secagem têm despertado o interesse de vários pesquisadores para os mais diferentes produtos: nozes de pistache (KASHANINEJAD et al., 2007), feijãovermelho (CORRÊA et al., 2007), semente de abóbora (SACILIK, 2007), tomate (DOYMAZ, 2007), sementes de amaranto (ABALONE et al., 2006), pimentavermelha (KALEEMULLAH; KAILAPPAN, 2006), trigo parboilizado (MOHAPATRA; RAO, 2005), milho (DOYMAZ; PALA, 2003), entre outros.

Uma vez que os modelos baseados na segunda Lei de Fick não são rigorosamente representativos dos diversos mecanismos que prevalecem no transporte de água em produtos agrícolas, e a difusividade varia conforme mudam as condições de secagem (temperatura e velocidade do ar), ou seja, não é intrínseca ao material, convenciona-se chamá-la de difusividade efetiva (ROCA et al., 2008).

Durante a modelagem e simulação dos processos de secagem de produtos agrícolas, diversos autores correlacionaram satisfatoriamente o coeficiente de difusão com os coeficientes dos modelos ajustados aos parâmetros de secagem, principalmente temperatura, umidade relativa e vazão do ar (DOYMAZ, 2005a; DOYMAZ, 2005b; MOHAPATRA; RAO, 2005).

Aenergia de ativação é definida como a facilidade com que as moléculas de água superam a barreira de energia durante a migração no interior do produto. Kayacier e Singh (2004) relatam que a energia de ativação diminui com a elevação do teor de água do produto. Nos processos de secagem, quanto menor a energia de ativação maior será a difusividade de água no produto. Corrêa et al. (2007) obtiveram uma energia de ativação para o feijão vermelho de 40,08 $\mathrm{KJ} \mathrm{mol}^{-1}$.
Considerando a importância do estudo teórico do processo de secagem dos produtos agrícolas e a limitação de informações teóricas a respeito dos fenômenos que ocorrem durante a secagem do feijão-caupi, este trabalho objetivou: a) estudar a cinética de secagem do feijãocaupi (Vigna unguiculata (L.) Walp.), b) ajustar diferentes modelos matemáticos aos valores experimentais em função do teor de água e, c) determinar o coeficiente de difusão e a energia de ativação dos grãos durante a secagem nas temperaturas de $25 ; 35 ; 45$ e $55^{\circ} \mathrm{C}$.

\section{MATERIAL E MÉTODOS}

Este trabalho foi desenvolvido no Laboratório de Secagem e Armazenagem de Produtos Vegetais do Curso de Engenharia Agrícola da Universidade Estadual de Goiás (UEG) em Anápolis-GO. Foram utilizados grãos de feijão-caupi (Vigna unguiculata (L.) Walp.), cultivar BRS-Maratoã, produzidas no município de Ipameri-GO na safra 2008/2009. Os grãos foram colhidos com teor de água de, aproximadamente, 27,8\% (b.s.), acondicionados em sacos plásticos e armazenados em refrigerador à temperatura de $4{ }^{\circ} \mathrm{C}$. Antes de cada experimento de secagem, as amostras foram retiradas do refrigerador e mantidas à temperatura ambiente, visando homogeneizar a temperatura. $\mathrm{O}$ teor de água inicial foi determinado pelo método-padrão da estufa, $105 \pm 3{ }^{\circ} \mathrm{C}$, durante $86400 \mathrm{~s}$ $(24 \mathrm{~h})$, com três repetições, de acordo com as Regras para Análise de Sementes (BRASIL, 2009).

A secagem do produto foi realizada nas temperaturas de $25 ; 35 ; 45$ e $55 \pm 1{ }^{\circ} \mathrm{C}$ em secador experimental (Figura 1) em três repetições.

As amostras foram colocadas em três bandejas removíveis (diâmetro de $0,09 \mathrm{~m}$ ) com fundo telado para permitir a passagem do ar através da massa do produto,

Figura 1 - Figura esquemática do secador experimental

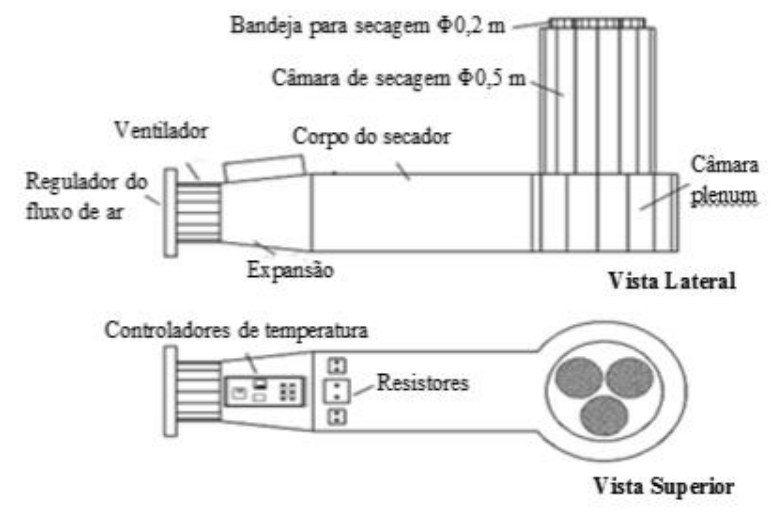


sendo consideradas as repetições. Cada bandeja continha, inicialmente, $0,26 \mathrm{~kg}$ de grãos de feijão-caupi, distribuídos homogeneamente em camadas finas.

A velocidade do ar foi monitorada com o auxílio de um anemômetro de lâminas rotativas e mantida constante em torno de $1,0 \mathrm{~m} \mathrm{~s}^{-1}$. A temperatura e a umidade relativa do ar de entrada foram monitoradas por meio de um psicrômetro giratório. Para o cálculo da temperatura e umidade relativa do ar de secagem foi utilizado o software Grapsi 5.1.

Durante o processo de secagem, as bandejas com as amostras foram pesadas periodicamente. A secagem dos grãos prosseguiu até o produto atingir o teor de água desejado, aproximadamente $13 \%$ b.s.

Para a determinação da razão de umidade dos grãos feijão-caupi para as diferentes condições de secagem, foi utilizada a Eq. 1. A umidade de equilíbrio foi calculada pelo modelo matemático de $\mathrm{GAB}$, com seus parâmetros determinados pelo processo de dessorção (MORAIS, 2010).

$$
R U=\frac{U-U_{e}}{U_{0}-U_{e}}
$$

Em que: RU é a razão de umidade (adimensional); U é o teor de água médio no tempo $\mathrm{t}$ (\% b.s.); Ue é o teor de água de equilíbrio (\% b.s.) e $\mathrm{U}_{0}$ é o teor de água inicial (\% b.s.).

Foram ajustados os modelos matemáticos, Tabela 1, aos dados experimentais de secagem. Para o ajuste foi utilizada análise de regressão não linear dos tipos polinomial de segundo grau e exponencial, por meio do software STATISTICA 6.0.
Foram estimados os valores dos parâmetros dos modelos, em função da variável independente tempo, para cada temperatura.

Em que: RU é a razão de umidade (adimensional); t é o tempo de secagem (h); k, $\mathrm{k}_{0}, \mathrm{k}_{1}$ são constantes de secagem $\left(h^{-1}\right)$; e a, b, c e n são coeficientes dos modelos.

$\mathrm{Na}$ seleção do melhor modelo para predizer a secagem das sementes de feijão-caupi foram considerados: a significância dos coeficientes de regressão, pelo teste t, ao nível de 5\% de significância; a magnitude do coeficiente de determinação ajustado $\left(\mathrm{R}^{2}\right)$, o erro relativo $(\mathrm{P})$ e o erro médio estimado (SE) (MOHAPATRA; RAO, 2005)

O coeficiente de difusão efetivo foi obtido por meio do ajuste do modelo matemático da difusão líquida, descrito pela Eq. 14, aos dados experimentais da secagem dos grãos de feijão-caupi. Essa equação é a solução analítica para a segunda lei de Fick, considerando a forma geométrica do produto como esférica, desconsiderando a contração volumétrica dos grãos e considerando a condição de contorno de teor de água conhecida na superfície do grão.

$$
R U=\frac{U-U_{e}}{U_{0}-U_{e}}=\frac{6}{\pi^{2}} \sum_{n=1}^{\infty} \frac{1}{n^{2}} \exp \left[-\frac{n^{2} \pi^{2} D_{e f} t}{r^{2}}\right]
$$

Em que: Def é o coeficiente de difusão efetivo $\left(\mathrm{m}^{2} \mathrm{~s}^{-1}\right)$; r é o raio equivalente $(\mathrm{m})$ e $\mathrm{n}$ é o número de termos.

O raio equivalente, utilizado no modelo da difusão, é definido como o raio de uma esfera cujo volume é igual ao do grão, sendo determinado por meio da medição dos três eixos ortogonais (comprimento, largura e espessura),

Tabela 1 - Modelos de regressão não-linear utilizados para predizer o fenômeno de secagem em camada delgada de grãos de feijão-caupi (Vigna unguiculata (L.) Walp.) cultivar BRS-Maratoã

\begin{tabular}{lcc}
\hline \multicolumn{1}{c}{ Nome } & Modelo & $\mathrm{N}^{\circ}$ \\
\hline Aproximação da difusão & $\mathrm{RU}=\mathrm{a} \exp (-\mathrm{Kt})+(1-\mathrm{a}) \exp (-\mathrm{Kbt})$ & $(2)$ \\
Dois termos & $\mathrm{RU}=\mathrm{a} \exp \left(-\mathrm{K}_{\mathrm{o}} \mathrm{t}\right)+\mathrm{bexp}\left(-\mathrm{K}_{1} \mathrm{t}\right)$ & $(3)$ \\
Exponencial de dois termos & $\mathrm{RU}=\mathrm{a} \exp (-\mathrm{Kt})+(1-\mathrm{a}) \exp (-\mathrm{Kat})$ & $(5)$ \\
Henderson e Pabis & $\mathrm{RU}=\mathrm{aexp}(-\mathrm{Kt})$ & $(6)$ \\
Henderson e Pabis modificado & $\mathrm{RU}=\mathrm{a} \exp (-\mathrm{Kt})+\mathrm{bexp}\left(-\mathrm{K}_{\mathrm{o}} \mathrm{t}\right)+\operatorname{cexp}\left(-\mathrm{K}_{1} \mathrm{t}\right)$ & $(7)$ \\
Logarítmico & $\mathrm{RU}=\mathrm{aexp}(-\mathrm{Kt})+\mathrm{c}$ & $(8)$ \\
Midilli & $\mathrm{RU}=\mathrm{aexp}\left(-\mathrm{K} \mathrm{t}^{\mathrm{n}}\right)+\mathrm{bt}$ & $(10)$ \\
Newton & $\mathrm{RU}=\exp (-\mathrm{Kt})$ & $(11)$ \\
Page & $\mathrm{RU}=\exp \left(-\mathrm{K} \mathrm{t}^{\mathrm{n}}\right)$ & $(12)$ \\
Thompson & $\mathrm{RU}=\exp \left(\left(-\mathrm{a}-\left(\mathrm{a}^{2}+4 \mathrm{bt}\right)^{0,5}\right) / 2 \mathrm{~b}\right)$ & $(13)$ \\
Verna & $\mathrm{RU}=\mathrm{a} \exp (-\mathrm{Kt})+(1-\mathrm{a}) \exp \left(-\mathrm{K}_{1} \mathrm{t}\right)$ & $\mathrm{RU}=1+\mathrm{at}+\mathrm{bt}{ }^{2}$ \\
Wang e Sing & & \\
\hline
\end{tabular}

Fonte: Doymaz (2004); Mohapatra e Rao (2005) 
em cinquenta grãos após a secagem, com auxílio de um paquímetro digital (precisão de $1.10^{-6} \mathrm{~m}$ ). O volume de cada grão (V), considerado como um esferóide triaxial (Figura 2), foi estimado utilizando-se a expressão 15:

$$
V=\frac{\pi(a \cdot b \cdot c)}{6}=\frac{4 \pi \cdot r^{3}}{3}
$$

Em que: a é o maior eixo do grão, comprimento (m); b é o eixo médio do grão, largura $(\mathrm{m})$ e c é o menor eixo do grão, espessura (m).

Figura 2 - Representação esquemática do esferóide triaxial de grãos do feijão-caupi

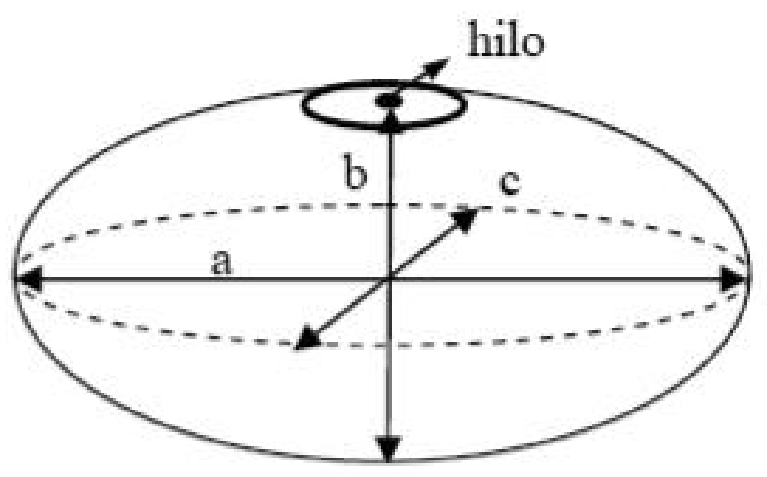

A variação do coeficiente de difusão de acordo com a temperatura de secagem foi analisada utilizando-se o modelo de Arrhenius, Eq. 16.

$V=\frac{\pi(a \cdot b \cdot c)}{6}=\frac{4 \cdot \pi \cdot r^{3}}{3}$

Em que: A é a constante $\left(\mathrm{m}^{2} \mathrm{~s}^{-1}\right)$; E é a energia de ativação $\left(\mathrm{J} \mathrm{mol}^{-1}\right)$; $\mathrm{R}$ é a constante universal dos gases $\left(8,314 \mathrm{~J} \mathrm{~mol}^{-1} \mathrm{~K}^{-1}\right)$ e $\mathrm{T}$ é a Temperatura absoluta $\mathrm{K}$.

\section{RESULTADOS E DISCUSSÃO}

No processo de determinação das curvas de secagem a umidade inicial dos grãos de feijão-caupi (Vigna unguiculata (L.) Walp.) foi de 27,8 $\pm 0,2 \%$ b.s.

$\mathrm{Na}$ Figura 3, são apresentados os valores experimentais da secagem de feijão-caupi para as temperaturas em estudo.

Analisando a Figura 3 verifica-se que o tempo necessário para o feijão-caupi atingir o teor de água de, aproximadamente 0,13 (b.s.), foi de 15,0; 10,7; 5,5 e 3,7 horas,
Figura 3 - Curvas experimentais de secagem de feijão-caupi (Vigna unguiculata (L.) Walp.) para as temperaturas de $25 ; 35 ; 45$ e $55^{\circ} \mathrm{C}$

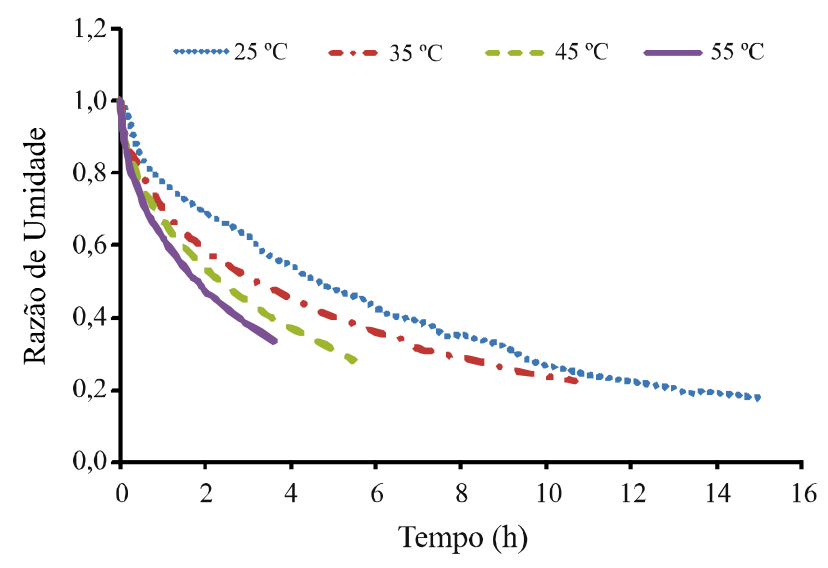

para as temperaturas de $25 ; 35 ; 45$ e $55^{\circ} \mathrm{C}$, respectivamente. Com o aumento da temperatura do ar de secagem, ocorreu maior taxa de remoção de água do produto, concordando com diversos pesquisadores para vários produtos agrícolas (BABALIS; BELESSIOTIS, 2004; LAHSASNI et al., 2004; MOHAPATRA; RAO, 2005).

Verifica-se também que a secagem dos grãos de feijão-caupi ocorre no período de taxa de secagem decrescente, indicando que ocorre uma maior resistência à transferência de calor e massa do interior do produto para sua superfície e, portanto, a difusão é o principal mecanismo que governa o movimento de água (KASHANINEJAD et al., 2007). Estes resultados estão de acordo com os trabalhos realizados sobre a secagem de diversos produtos, como milho pipoca (CORRÊA; MACHADO; ANDRADE, 2001), trigo (MOHAPATRA; RAO, 2005) e feijão (RESENDE et al., 2007).

Nas Tabelas 2; 3; 4 e 5, são mostrados os coeficientes de cada modelo matemático na modelagem das curvas de secagem do feijão-caupi nas temperaturas $25 ; 35 ; 45$ e $55^{\circ} \mathrm{C}$.

$\mathrm{Na}$ Tabela 6, são apresentados os valores dos coeficientes de determinação, erros médios, relativo e estimado, para os doze modelos ajustados para a secagem do feijão-caupi (Vigna unguiculata (L.) Walp.) nas temperaturas de $25 ; 35 ; 45$ e $55^{\circ} \mathrm{C}$.

$\mathrm{Na}$ Tabela 6 observa-se que nas quatro temperaturas utilizadas de secagem do feijão-caupi, os doze modelos matemáticos ajustados aos dados experimentais apresentaram coeficientes de determinação $\left(\mathrm{R}^{2}\right)$ superiores a 90\% (Tabela 6), indicando, de acordo com Mohapatra e Rao (2005), uma representação satisfatória do processo de secagem. Nota-se também, que entre os que apresentaram 
Tabela 2 - Coeficientes dos modelos matemáticos ajustados da curva de secagem do feijão-caupi (Vigna unguiculata (L.) Walp.) BRS-Maratoã para a temperatura $25^{\circ} \mathrm{C}$

\begin{tabular}{cccccccc}
\hline \multirow{2}{*}{ Modelo } & \multicolumn{7}{c}{ Coeficientes } \\
\cline { 2 - 7 } & $\mathrm{a}$ & $\mathrm{b}$ & $\mathrm{c}$ & $\mathrm{n}$ & $\mathrm{k}$ & $\mathrm{k}_{0}$ & $\mathrm{k}_{1}$ \\
\hline$(2)$ & 0,1459 & 0,0540 & - & - & 2,0700 & - & - \\
$(3)$ & 0,1649 & 0,8569 & - & - & - & 2,4628 & 0,1121 \\
$(4)$ & 0,1319 & - & - & - & 0,8891 & - & - \\
$(5)$ & 0,9121 & - & - & - & 0,1219 & - & - \\
$(6)$ & 0,1581 & 0,000004 & 0,8649 & - & 2,6820 & $-0,5856$ & 0,1149 \\
$(7)$ & 0,8219 & - & 0,1119 & - & 0,1632 & - & - \\
$(8)$ & 1,0105 & $-0,0055$ & - & 0,6632 & 0,2368 & - & - \\
$(9)$ & - & - & - & - & 0,1394 & - & - \\
$(10)$ & - & - & - & 0,7531 & 0,2239 & - & - \\
$(11)$ & $-1,9273$ & 0,6888 & - & - & - & - & - \\
$(12)$ & 0,8541 & - & - & - & 0,1118 & - & 2,0700 \\
$(13)$ & $-0,1283$ & 0,0052 & - & - & - & - & - \\
\hline
\end{tabular}

Tabela 3 - Coeficientes dos modelos matemáticos ajustados da curva de secagem do feijão-caupi (Vigna unguiculata (L.) Walp.) BRSMaratoã para a temperatura $35^{\circ} \mathrm{C}$

\begin{tabular}{cccccccc}
\hline \multirow{2}{*}{ Modelo } & \multicolumn{7}{c}{ Coeficientes } \\
\cline { 2 - 8 } & $\mathrm{a}$ & $\mathrm{b}$ & $\mathrm{c}$ & $\mathrm{n}$ & $\mathrm{k}$ & $\mathrm{k}_{0}$ & $\mathrm{k}_{1}$ \\
\hline$(2)$ & 0,2741 & 0,0686 & - & - & 1,6778 & - & - \\
$(3)$ & 0,2614 & 0,7122 & - & - & - & 1,3716 & 0,1124 \\
$(4)$ & 0,1550 & - & - & - & 1,0075 & - & - \\
$(5)$ & 0,8527 & - & - & - & 0,1475 & - & - \\
$(6)$ & 0,0669 & 0,6886 & 0,2460 & - & 11,7916 & 0,1079 & 1,0060 \\
$(7)$ & 0,6849 & - & 0,2243 & - & 0,2985 & - & - \\
$(8)$ & 1,0070 & $-0,0023$ & - & 0,5901 & 0,3473 & - & - \\
$(9)$ & - & - & - & - & 0,1936 & - & - \\
$(10)$ & - & - & - & 0,6186 & 0,3418 & - & - \\
$(11)$ & $-0,5457$ & 0,6186 & - & - & - & - & - \\
$(12)$ & 0,7259 & - & - & - & 0,1152 & - & - \\
$(13)$ & $-0,1915$ & 0,0121 & - & - & - & - \\
\hline
\end{tabular}

Tabela 4 - Coeficientes dos modelos matemáticos ajustados da curva de secagem do feijão-caupi (Vigna unguiculata (L.) Walp.) BRSMaratoã para a temperatura $45^{\circ} \mathrm{C}$

\begin{tabular}{cccccccc}
\hline \multirow{2}{*}{ Modelo } & \multicolumn{7}{c}{ Coeficientes } \\
\cline { 2 - 7 } & $\mathrm{a}$ & $\mathrm{b}$ & $\mathrm{c}$ & $\mathrm{n}$ & $\mathrm{k}$ & $\mathrm{k}_{0}$ & $\mathrm{k}_{1}$ \\
\hline$(2)$ & 0,1978 & 0,0542 & - & - & 3,5794 & - & - \\
$(3)$ & 0,1885 & 0,7922 & - & - & - & 2,8920 & 0,1900 \\
$(4)$ & 0,1331 & - & - & - & 1,7669 & - & - \\
$(5)$ & 0,8787 & - & - & - & 0,2290 & - & - \\
$(6)$ & 0,0743 & 0,7544 & 0,1704 & - & 16,1969 & 0,1771 & 1,5235 \\
\hline
\end{tabular}


Continuação da Tabela 4

\begin{tabular}{rccccccc}
\hline$(7)$ & 0,6653 & - & 0,2555 & - & 0,4450 & - & - \\
$(8)$ & 0,9989 & $-0,0124$ & - & 0,5879 & 0,3818 & - & - \\
$(9)$ & - & - & - & - & 0,2911 & - & - \\
$(10)$ & - & - & - & 0,6438 & 0,4033 & - & - \\
$(11)$ & $-0,5522$ & 0,6896 & - & - & - & - & - \\
$(12)$ & 0,1978 & - & - & - & 3,5796 & - & 0,1939 \\
$(13)$ & $-0,3042$ & 0,0344 & - & - & - & - & - \\
\hline
\end{tabular}

Tabela 5 - Coeficientes dos modelos matemáticos ajustados da curva de secagem do feijão-caupi (Vigna unguiculata (L.) Walp.) BRSMaratoã para a temperatura $55^{\circ} \mathrm{C}$

\begin{tabular}{|c|c|c|c|c|c|c|c|}
\hline \multirow{2}{*}{ Modelo } & \multicolumn{7}{|c|}{ Coeficientes } \\
\hline & $\mathrm{a}$ & b & c & $\mathrm{n}$ & $\mathrm{k}$ & $\mathrm{k}_{0}$ & $\mathrm{k}_{1}$ \\
\hline (2) & 0,2093 & 0,0592 & - & - & 4,1283 & - & - \\
\hline (3) & 0,7848 & 0,2041 & - & - & - & 0,2415 & 3,6996 \\
\hline (4) & 0,1317 & - & - & - & 2,3530 & - & - \\
\hline (5) & 0,8817 & - & - & - & 0,3028 & - & - \\
\hline (6) & 0,1008 & 0,6980 & 0,2007 & - & 10,0018 & 0,2028 & 1,3484 \\
\hline (7) & 0,6212 & - & 0,3167 & - & 0,7174 & - & - \\
\hline (8) & 1,0012 & $-0,0066$ & - & 0,6086 & 0,4661 & - & - \\
\hline (9) & - & - & - & - & 0,3867 & - & - \\
\hline (10) & - & - & - & 0,6314 & 0,4773 & - & - \\
\hline (11) & $-0,4462$ & 0,7352 & - & - & - & - & - \\
\hline (12) & 0,7907 & - & - & - & 0,2446 & - & 4,1286 \\
\hline (13) & $-0,4302$ & 0,0739 & - & - & - & & \\
\hline
\end{tabular}

Tabela 6 - Coeficientes de determinação $\left(\mathrm{R}^{2}, \%\right)$, erros médio relativo (P, \%), estimado (SE, decimal) para os doze modelos analisados, para a secagem do feijão-caupi (Vigna unguiculata (L.) Walp.) cultivar BRS-Maratoã nas temperaturas $25 ; 35 ; 45$ e $55^{\circ} \mathrm{C}$

\begin{tabular}{|c|c|c|c|c|c|c|c|c|c|c|c|c|}
\hline \multirow{2}{*}{$\mathrm{N}^{\mathrm{o}}$} & \multicolumn{3}{|c|}{$25^{\circ} \mathrm{C}$} & \multicolumn{3}{|c|}{$35^{\circ} \mathrm{C}$} & \multicolumn{3}{|c|}{$45^{\circ} \mathrm{C}$} & \multicolumn{3}{|c|}{$55^{\circ} \mathrm{C}$} \\
\hline & $\mathrm{R}^{2}$ & $\mathrm{P}$ & SE & $\mathrm{R}^{2}$ & $\mathrm{P}$ & SE & $\mathrm{R}^{2}$ & $\mathrm{P}$ & SE & $\mathrm{R}^{2}$ & $\mathrm{P}$ & SE \\
\hline (2) & 99,93 & 2,07 & 0,01 & 99,91 & 1,29 & 0,01 & 99,92 & 1,08 & 0,01 & 99,94 & 0,92 & 0,01 \\
\hline (3) & 99,94 & 2,03 & 0,01 & 99,94 & 0,96 & 0,01 & 99,94 & 0,89 & 0,01 & 99,94 & 0,86 & 0,01 \\
\hline (4) & 99,55 & 3,89 & 0,02 & 97,17 & 10,23 & 0,05 & 97,95 & 5,94 & 0,04 & 97,68 & 5,77 & 0,04 \\
\hline (5) & 99,45 & 4,28 & 0,03 & 98,25 & 6,82 & 0,04 & 98,68 & 4,11 & 0,03 & 98,20 & 4,32 & 0,03 \\
\hline (6) & 99,95 & 1,37 & 0,01 & 99,98 & 0,73 & 0,00 & 99,99 & 0,31 & 0,00 & 99,99 & 0,31 & 0,00 \\
\hline (7) & 99,65 & 2,86 & 0,02 & 99,44 & 3,76 & 0,02 & 99,49 & 2,51 & 0,02 & 99,49 & 2,27 & 0,02 \\
\hline (8) & 99,87 & 2,48 & 0,01 & 99,97 & 0,90 & 0,01 & 100,00 & 0,27 & 0,00 & 99,99 & 0,33 & 0,00 \\
\hline (9) & 98,10 & 9,79 & 0,05 & 92,81 & 16,47 & 0,08 & 93,86 & 0,07 & 0,07 & 93,06 & 10,15 & 0,07 \\
\hline (10) & 99,83 & 2,83 & 0,02 & 99,96 & 1,13 & 0,01 & 99,96 & 0,89 & 0,01 & 99,99 & 0,42 & 0,00 \\
\hline (11) & 99,62 & 4,73 & 0,02 & 99,89 & 2,18 & 0,01 & 99,79 & 1,97 & 0,01 & 99,91 & 1,11 & 0,01 \\
\hline (12) & 99,93 & 2,07 & 0,01 & 99,91 & 1,29 & 0,01 & 99,92 & 1,08 & 0,01 & 99,94 & 0,92 & 0,01 \\
\hline (13) & 97,81 & 9,57 & 0,05 & 93,98 & 13,81 & 0,08 & 95,48 & 8,68 & 0,06 & 96,06 & 7,40 & 0,05 \\
\hline
\end{tabular}


melhores valores para os parâmetros estatísticos para cada temperatura o modelo de Henderson e Pabis modificada é o mais recomendado, apresentando: $\mathrm{R}^{2}=99,95 \%, \mathrm{P}=1,37 \%$ e $\mathrm{SE}=0,0082$ para $25^{\circ} \mathrm{C} ; \mathrm{R}^{2}=99,97 \%, \mathrm{P}=0,73 \%$ e $\mathrm{SE}=0,0048$, para $35{ }^{\circ} \mathrm{C} ; \mathrm{R}^{2}=99,99 \%, \mathrm{P}=0,2696 \%$ e $\mathrm{SE}=0,00179$ para $45{ }^{\circ} \mathrm{C}$ e; $\mathrm{R}^{2}=99,99 \%, \mathrm{P}=0,3135 \%$, $\mathrm{SE}=0,00265$ para $55^{\circ} \mathrm{C}$. O modelo de Midilli também representa satisfatoriamente a secagem nas temperaturas de 45 e $55^{\circ} \mathrm{C}$.

Na Figura 4 são mostradas as curvas de secagem de feijão-caupi experimentais e, estimadas pelo modelo matemático de Henderson de Pabis Modificado em função do tempo para as temperaturas em estudo.

Figura 4 - Curvas de secagem estimadas e experimentais de feijão-caupi (Vigna unguiculata (L.) Walp.) nas temperaturas $25 ; 35 ; 45$ e $55^{\circ} \mathrm{C}$

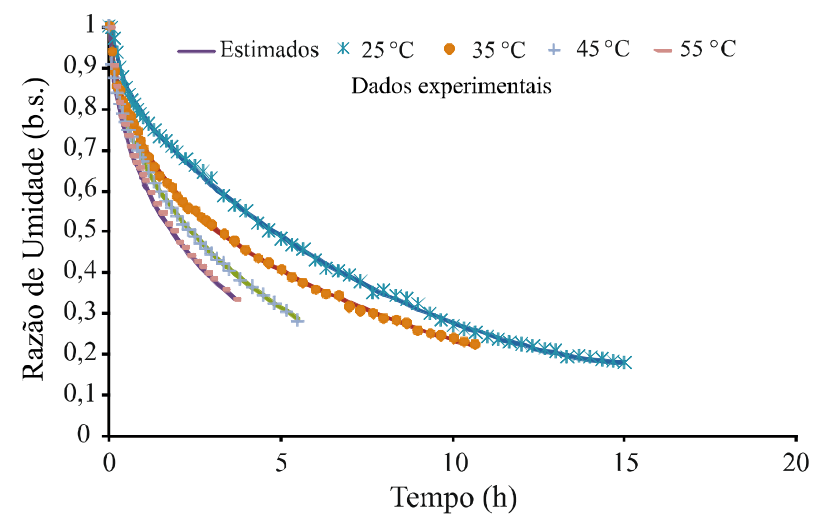

Na Tabela 7, são apresentados os valores do coeficiente de difusão obtidos durante a secagem do feijão nas diferentes condições de temperatura, utilizando o raio equivalente de $0,0035 \mathrm{~m}$. Os dados foram obtidos pelo ajuste da Eq. 14 aos dados experimentais de secagem de feijão-caupi, com aproximação de oito termos, a partir do qual se observou que o valor de Def não variava.
Nota-se que com a elevação da temperatura, os valores do coeficiente de difusão aumentaram sensivelmente, assim como mostram os resultados relatados por Doymaz (2005b) para quiabo, que variaram entre 4,27 × $10^{-10}$ a $13,0 \times 10^{-10} \mathrm{~m}^{2} \mathrm{~s}^{-1}$ para a faixa de temperatura entre 50 e $70{ }^{\circ} \mathrm{C}$; Panchariya, Popovic e Sharma (2002) na secagem de chá preto, obtiveram valores entre $1,141 \times 10^{-11}$ a $2,985 \times 10^{-11} \mathrm{~m}^{2} \mathrm{~s}^{-1}$ para a faixa de temperatura de 80 a $120{ }^{\circ} \mathrm{C}$. Akpinar (2006) obteve valores entre $4,53 \times 10^{-12}$ a $7,04 \times 10^{-12} \mathrm{~m}^{2} \mathrm{~s}^{-1}$ para as espécies menta (Mentha sp.), salsinha (Petroselinum crispum) e manjericão (Ocimum basilicum).

Na Tabela 8, são mostrados os coeficientes da regressão linear dos valores do coeficiente de difusão efetivo (Def) em função da temperatura para o feijãocaupi.

Tabela 8 - Coeficientes (a e b) e parâmetros estatísticos da regressão linear para o Def do feijão-caupi (Vigna unguiculata (L.) Walp.) BRS-Maratoã em função da temperatura

\begin{tabular}{ll}
\hline \multicolumn{1}{c}{$\mathrm{a}$} & $\mathrm{b}$ \\
\hline $3,7761.10-9$ & $-5,9487.10^{-9}$ \\
$\mathrm{R}^{2}(\%)$ & 99,0 \\
$\mathrm{P}(\%)$ & 3,038 \\
$\mathrm{SE}$ (decimal) & $8,08.10^{-8}$ \\
\hline
\end{tabular}

Durante a secagem dos grãos de feijão-caupi, os coeficientes de difusão apresentaram magnitudes entre $8,84 \times 10^{-8}$ e $20,17 \times 10^{-8} \mathrm{~m}^{2} \mathrm{~s}^{-1}$, para a faixa de temperatura de 25 a $55^{\circ} \mathrm{C}$.

Os valores calculados de Def estão apresentados, também, na Figura 5, na forma de "InDef", descritas em função do recíproco da temperatura absoluta (1/T). A reta obtida indica a uniformidade de variação da difusividade com a variação da temperatura.

Tabela 7 - Coeficiente de difusão e parâmetros estatísticos para as temperaturas de $25 ; 35 ; 45$ e $55{ }^{\circ} \mathrm{C}$

\begin{tabular}{ccccc}
\hline Temperatura & Def $\left(\times 10^{-8}\right)$ & $\mathrm{R}^{2}(\%)$ & $\mathrm{P}(\%)$ & SE $($ decimal $)$ \\
\hline $25^{\circ} \mathrm{C}$ & 8,84 & 0,9856 & 9,0760 & 0,0437 \\
$35^{\circ} \mathrm{C}$ & 11,66 & 0,9972 & 1,5417 & 0,0168 \\
$45^{\circ} \mathrm{C}$ & 15,78 & 0,9942 & 2,7743 & 0,0211 \\
$55^{\circ} \mathrm{C}$ & 20,17 & 0,9938 & 2,2787 & 0,0208 \\
\hline
\end{tabular}


Figura 5 - Representação de Arrhenius para a relação entre a difusividade efetiva e a temperatura absoluta para os grãos de feijão-caupi (Vigna unguiculata (L.) Walp.) cultivar BRSMaratoã

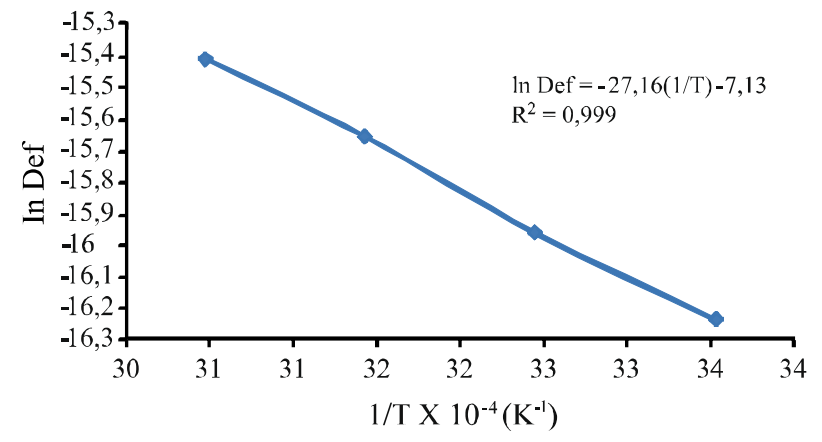

A energia de ativação (E) para a difusão líquida do feijão, calculada como a inclinação da reta obtida do $\ln$ (Def), foi de 27,16 kJ mol${ }^{-1}$, valor que corresponde aos encontrados na literatura especializada, para diversos produtos agrícolas: Arroz cozido, $36,4 \mathrm{~kJ} \mathrm{~mol}^{-1}$ (RAMESH, 2003); cenoura, 28,36 kJ mol-1 (DOYMAZ, 2004); milho da espécie Zea mays indentata, $29,56 \mathrm{~kJ} \mathrm{~mol}^{-1}$ (DOYMAZ; PALA, 2003); grãos de mamona, 15,25 kJ $\mathrm{mol}^{-1}$ (GONELLI, 2008); feijão vermelho, 40,08 $\mathrm{kJ} \mathrm{mol}^{-1}$ (RESENDE et al., 2007); folhas de capim limão, 63,47 kJ mol $^{-1}$ (MARTINAZZO et al., 2007); Nozes de pistachio, $30,79 \mathrm{~kJ} \mathrm{~mol}^{-1}$ (KASHANINEJAD et al., 2007); Semente de abóbora, 33,15 kJ mol ${ }^{-1}$ (SACILIK, 2007); Semente de quinoa, 37,98 kJ mol ${ }^{-1}$ (GELY; SANTALLA, 2007); Soja (secas abaixo de $50{ }^{\circ} \mathrm{C}$ ), 16,60 $\mathrm{kJ} \mathrm{mol}^{-1}$ (GELY; GINER, 2007); Soja (secas acima de $50^{\circ} \mathrm{C}$ ), 28, $80 \mathrm{~kJ} \mathrm{~mol}^{-1}$ (GELY; GINER, 2007); Trigo, 42,00 kJ mol-1 (GONELI et al., 2007); Arroz em casca, 34,80 $\mathrm{kJ} \mathrm{mol}^{-1}$ (RESENDE et al., 2005); Trigo parboilizado, 27,01 kJ mol ${ }^{-1}$ (MOHAPATRA; RAO, 2005); e para o feijão-verde, 39,47 $\mathrm{kJ} \mathrm{mol}^{-1}$ (SENADEERA et al., 2003) e $35,43 \mathrm{~kJ} \mathrm{~mol}^{-1}$ (DOYMAZ, 2005a).

\section{CONCLUSÕES}

1.O tempo necessário para a secagem do feijão-caupi até o teor de água na faixa de 0,11 a 0,13 (decimal b.s.) foi de $15 ; 10,66 ; 5,5$ e 3,66 horas, para as temperaturas de $25 ; 35 ; 45$ e $55^{\circ} \mathrm{C}$, respectivamente, evidenciando que o tempo requerido para a secagem do feijão-caupi depende, da temperatura de secagem;

2.O modelo matemático de Henderson e Pabis Modificada, utilizada para caracterizar a secagem, foi o que melhor representou o processo de secagem do feijão-caupi, ajustando-se satisfatoriamente aos dados experimentais;

3.O coeficiente de difusão aumenta com a elevação da temperatura, apresentando valores entre $8,84 \times 10^{-8} \mathrm{e}$ $20,17 \times 10^{-8} \mathrm{~m}^{2} \mathrm{~s}^{-1}$, para a faixa de temperatura de 25 a $55^{\circ} \mathrm{C}$, respectivamente;

4. A relação do coeficiente de difusão com a temperatura de secagem pode ser descrita pela equação de Arrhenius, que apresenta uma energia de ativação de $27,16 \mathrm{~kJ} \mathrm{~mol}^{-1}$ para a difusão líquida no processo de secagem dos grãos de feijão-caupi.

\section{AGRADECIMENTOS}

À Capes, pelo apoio financeiro e à pesquisa, por meio dos recursos dos programas Especial e Procad.

\section{REFERÊNCIAS}

ABALONE, R. et al. Thin layer drying of amaranth seeds. Biosystems Engineering, v. 93, n. 2, p. 179-188, 2006.

AKPINAR, E. K. Mathematical modelling of thin layer drying process under open sun of some aromatic plants. Journal of Food Engineering, v. 77, n. 4, p. 864-870, 2006.

BABALIS, S. J.; BELESSIOTIS, V. G. Influence of the drying conditions on the drying constants and moisture diffusivity during the thin-layer drying of figs. Journal of Food Engineering, v. 65, n. 3, p. 449-458, 2004.

BORÉM, A.; CARNEIRO, J. E. S. A cultura. In: VIEIRA, C.; PAULA JÚNIOR, T. J.; BORÉM, A. Feijão. 2. ed. Viçosa: UFV, 2006. p. 15-18.

BRASIL. Ministério da Agricultura e Reforma Agrária. Secretaria Nacional de defesa Agropecuária. Regras para análise de sementes. Brasília, 2009. 365 p.

CORRÊA, P. C.; MACHADO, P. F.; ANDRADE, E. T. de. Cinética de secagem e qualidade de grãos de milho-pipoca. Revista Ciências e Agrotecnologia, v. 25, n. 1, p. 134-142, 2001.

CORRÊA, P. C. et al. Modelagem matemática para a descrição do processo de secagem do feijão (phaseolusvulgarisL.) em camadas delgadas. Engenharia Agrícola, v. 27, n. 2, p. 501507, 2007.

DOYMAZ, I.; PALA, M. The thin-layer drying characteristics of corn. Journal of Food Engineering, v .60, n. 2, p. 125-130, 2003.

DOYMAZ, I. Convective air drying characteristics of thin layer carrots. Journal of Food Engineering, v. 61, n. 3, p. 359-364, 2004.

DOYMAZ, I. Drying behaviour of green beans. Journal of Food Engineering, v. 69, n. 2, p. 161-165, 2005a. 
DOYMAZ, I. Drying characteristics and kinects of ockra. Journal of Food Engineering, v. 69, n. 3, p. 275-279, 2005b.

DOYMAZ, I. Air drying characteristics of tomatoes. Journal of Food Engineering, v. 78, n. 4, p. 1291-1297, 2007.

GELY, M. C.; GINER, S. A. Diffusion coefficient relationships during drying of soya bean cultivars. Biosystems Engineering, v. 96, p. 213-222, 2007.

GELY, M. C.; SANTALLA, E. M. Moisture diffusivity in quinoa (Chenopodiun quinoa Willd.) sedds: Effect of air temperature and initial moisture content of seeds. Journal of Food Engineering, v. 78, p. 1029-1033, 2007.

GONELI, A. L. D. et al. Estudo da difusão de umidade em grãos de trigo durante a secagem. Ciência e Tecnologia de Alimentos, v. 27, n. 1, p. 135-140, 2007.

GONELI, A. L. D. Variação das propriedades físicasmecânicas e da qualidade da mamona (Ricinus communis L.) durante a secagem e o armazenamento. 2008. $186 \mathrm{f}$. Tese (Doutorado em Engenharia Agrícola) - Universidade Federal de Viçosa, Viçosa, 2008.

KALEEMULLAH, S.; KAILAPPAN, R. Modelling of thin-layer drying kinetics of red chillies. Journal of Food Engineering, v. 76, p. 531-537, 2006.

KASHANINEJAD, M. et al. Thin-layer drying characteristics and modeling of pistachio nuts. Journal of Food Engineering, v. 78, n. 1, p. 98-108, 2007.

KAYACIER, A.; SINGH, R. K. Application of effetive diffusivity approach for the moisture content prediction of tortilla chips during baking. Swiss Society of Food Science and Technology, v. 37 , n. 2 , p. $275-281,2004$.

LAHSASNI, S. et al. Drying kinetcs of prickly pear fruit (Opuntiaficusindica). Journal of Food Engineering, v. 61, n. 2, p. 173-179, 2004.
MARTINAZZO, A. P. et al. Análise e descrição matemática da cinética de secagem de folhas de capim-limão. Revista Brasileira de Engenharia Agrícola e Ambiental, v. 11, n. 3, p. 301-306, 2007.

MOHAPATRA, D.; RAO, P. S.A thin layer drying model of parboiled wheat. Journal of Food Engineering, v. 66, n. 4, p. 513-518, 2005.

MORAIS, S. J. M. Cinética de secagem de grãos de feijãocaupi. 2010. 90 f. Dissertação (Mestrado em Engenharia Agrícola) - Universidade Estadual de Goiás, Goiás, 2010.

PANCHARIYA, P. C.; POPOVIC, D.; SHARMA, A. L. Thinlayer modeling of Black tea drying process. Journal of Food Engineering, v. 52, n. 4, p. 349-357, 2002.

RAMESH, M. N. Moisture transfer properties of cooked rice during drying. Journal of Food Science, v. 36, n. 2, p. $245-255,2003$.

RESENDE, O. et al. Contração volumétrica na difusão líquida durante o processo de secagem do arroz em casca. Revista Brasileira de Armazenamento, v. 30, p. 163-171, 2005.

RESENDE, O. et al. Bean moisture diffusivity and drying kinetics: a comparison of the liquid diffusion model when taking into account and neglecting grain shrinkage. Spanish Journal of Agricultural Research, v. 05, p. 51-58, 2007.

ROCA, E. et al. Effective moisture diffusivity modeling food structure and hygroscopicity. Food Chemistry, v. 106, n. 4, p. 1428-1437, 2008.

SACILIK, K. Effect of drying methods on thin-layer drying characteristics of hull-less pumpkin (Cucurbita pepo L.). Journal of Food Engineering, v. 79, p. 23-30, 2007.

SENADEERA, W. et al. Influence of shapes of selected vegetable materials on drying kinetics during fluidized bed drying. Journal of Food Engineering, v. 58, n. 3, p. 277-283, 2003. 